\title{
Wnt2 overexpression protects against PINK1 mutant-induced mitochondrial dysfunction and oxidative stress
}

\author{
SUI-RUI XIA ${ }^{1,2^{*}}$, XUE-YI WEN $^{3 *}$, XIAO-LI FAN ${ }^{2}$, XIAO-RONG CHEN ${ }^{2}$, \\ ZAI-WA WEI ${ }^{2,3}$, QING-HUA LI ${ }^{3-6}$ and LI SUN ${ }^{2}$ \\ ${ }^{1}$ Department of Hospital Infection-Control, Nanxishan Hospital of Guangxi Zhuang Autonomous Region, Guilin, \\ Guangxi 541002; ${ }^{2}$ Faculty of Basic Medical Sciences; ${ }^{3}$ Guangxi Key Laboratory of Brain and Cognitive Neuroscience, \\ Guilin Medical University, Guilin, Guangxi 541004; ${ }^{4}$ Department of Neurology; ${ }^{5}$ Guangxi Clinical Research \\ Center for Neurological Disease; ${ }^{6}$ Laboratory of Neuroscience, Affiliated Hospital of \\ Guilin Medical University, Guilin, Guangxi 541004, P.R. China
}

Received July 21, 2019; Accepted March 12, 2020

DOI: $10.3892 / \mathrm{mmr} .2020 .11066$

\begin{abstract}
The PTEN induced putative kinase 1 (PINK1) mutation is the second most common cause of autosomal recessive adolescent Parkinson's disease (PD). Furthermore, mitochondrial disorders and oxidative stress are important mechanisms in the pathogenesis of PD. Numerous members of the Wnt family have been found to be associated with neurodegenerative diseases. Therefore, the present study investigated the role of the Wnt 2 gene in $P I N K 1^{B 9}$ transgenic flies, which is a PD model, and its underlying mechanism. It was identified that overexpression of Wnt2 reduced the abnormality rate of PD transgenic Drosophila and improved their flight ability, while other intervention groups had no significant effect. Furthermore, an increase in ATP concentration normalized mitochondrial morphology, and increased the mRNA expression levels of NADH-ubiquinone oxidoreductase chain 1 (NDI), ND42, ND75, succinate dehydrogenase complex subunits B, Cytochrome $\mathrm{b}$ and Cyclooxygenase 1 , which are associated with Wnt2 overexpression. Moreover, overexpression of Wnt2 in PD transgenic Drosophila resulted in the downregulation of reactive oxygen species and malondialdehyde production, and increased manganese superoxide dismutase (MnSOD), while
\end{abstract}

Correspondence to: Professor Qing-Hua Li, Guangxi Key Laboratory of Brain and Cognitive Neuroscience, Guilin Medical University, 109 North 2nd Huancheng Road, Guilin, Guangxi 541004, P.R. China

E-mail: qhli1999@163.com

Professor Li Sun, Faculty of Basic Medical Sciences, Guilin Medical University, 109 North 2nd Huancheng Road, Guilin, Guangxi 541004, P.R. China

E-mail: sunli2196@glmc.edu.cn

*Contributed equally

Key words: PTEN induced putative kinase 1, mitochondria, oxidative stress, Wnt2, PPARG coactivator $1 \alpha$, forkhead box sub-group $O$ glutathione was not significantly affected. It was found that overexpression of $\mathrm{Wnt} 2$ did not alter the protein expression of $\beta$-catenin in $P I N K 1^{\mathrm{B} 9}$ transgenic Drosophila, but did increase the expression levels of PPARG coactivator $1 \alpha(P G C-1 \alpha)$ and forkhead box sub-group $\mathrm{O}(F O X O)$. Collectively, the present results indicated that the $W n t 2$ gene may have a protective effect on PD PINK $1^{B 9}$ transgenic Drosophila. Thus, it was speculated that the reduction of oxidative stress and the restoration of mitochondrial function via Wnt2 overexpression may be related to the PGC- $1 \alpha / \mathrm{FOXO} / \mathrm{MnSOD}$ signaling pathway in PINK1 mutant transgenic Drosophila.

\section{Introduction}

PTEN-induced kinase 1 (PINK1) is located in the mitochondrial membrane, and helps to regulate mitochondrial morphology and autophagy (1). Mutations in PINK1 have been recognized to be the second most common cause of autosomal recessive adolescent Parkinson's disease (PD) (2). Loss of Drosophila PINK1 leads to mitochondrial morphological disorders and mitochondrial complex function damage (3). Furthermore, neurons are dependent on mitochondria, which act as the major energy producers (4). Therefore, when PINKI is mutated, neurotoxicity is increased and this may be associated with mitochondrial defects, thus contributing to the loss of dopaminergic (DA) neurons (5). Oxidative stress is a prominent and common feature of all forms of PD, and may have a toxic effect that causes neuronal cell death (6). Moreover, oxidative stress in PD is closely associated with a series of pathogenic factors, including mitochondrial dysfunction, DA metabolism and metal ion dysregulation (7).

The human Wnt gene family, known as the wingless-type MMTV integration site family, consists of 19 members, and the interaction between Wntl and Wnt5a promotes the development of DA neurons in the midbrain (8). Moreover, the Wnt 2 gene is one of the 19 Wnt family members that is highly expressed in the human thalamus, and plays an important role in the late development of human genes and the brain $(9,10)$. A previous functional study has shown that Wnt2 promotes the migration of primitive neurons and increases the number of 
DA neurons (11), thus enhancing DA function in the midbrain, and affecting mRNA and protein expression levels. The Wnt signaling pathway can be cross-linked with a number of signaling pathways (12). The interaction of $\beta$-catenin with the forkhead box sub-group O (FOXO) signaling pathway can inhibit Huntington protein toxicity (13). Furthermore, the Wnt signaling pathway also regulates mitochondrial energy, metabolism and oxidative stress $(14,15)$.

The present study selected Drosophila as the experiment model, as Drosophila genes have been fully sequenced and annotated, and are highly homologous to human genes $(16,17)$. Compared with other models, the Drosophila life cycle is very short; therefore, it is easier to observe the whole process of disease development (18). Mature genetic systems, abundant strain resources and powerful genome editing techniques also make Drosophila one of the primary choices for genetic research in neurodegenerative diseases (19).

The present study identified that overexpression of Wnt2 gene had a significant effect on PINK $1^{B 9}$ transgenic Drosophila. While the Wnt pathway may have neuroprotective effects, the role of Wnt 2 in neurodegenerative diseases remains unknown. Therefore, the aims of the present study were to investigate the function of Wnt2 in PINK1 mutant transgenic Drosophila, and to identify its association with the Wnt/ $\beta$-catenin and PPARG coactivator $1 \alpha(\mathrm{PGC}-1 \alpha) / \mathrm{FOXO} / \mathrm{manganese}$ superoxide dismutase (MnSOD) signaling pathways.

\section{Materials and methods}

Drosophila stocks. A total of five fly stocks were used: two stocks of Drosophila melanogaster (UAS-Wnt2OE and UAS-Wnt2RNAi) were purchased from the Bloomington Drosophila Stock Center. In total, three stocks (UAS-PINK1 $1^{B 9} / \mathrm{FM} 7$; MHC-Gal4, W1118 and MHC-GAL4) were provided by Institute of Life Sciences of Fuzhou University. W1118 is a wild-type genotype. UAS-Wnt2OE is a stock that overexpresses the Wnt2 gene. UAS-Wnt2RNAi is a Drosophila that has lost the function of the Wnt2 gene by using RNA interference technology. MHC-GAL4 is a Drosophila with an indirect flying muscle promoter. UAS-PINK1 $1^{B 9} / \mathrm{FM} 7$; MHC-Gal4 is a PD Drosophila model, in which the PINK1 mutation gene can be specifically expressed in indirect flying muscles. The classic GAL4/UAS system is divided into two parts: GAL4 and UAS. The GAL4 stock and UAS stock are two independent stocks (20). The fusion of GAL4 and tissue-specific promoter can regulate the expression of GAL4 protein in different tissues of Drosophila (21). Furthermore, UAS and target genes are fused to construct a transgenic line with a UAS-target gene (22). Only when the two hybridize, can GAL4 recognize the UAS promoter and induce expression of UAS downstream genes in specific tissues (23). Drosophila were placed in Drosophila culture tubes containing corn medium and cultured at a constant temperature of $25^{\circ} \mathrm{C}$ and $60 \%$ relative humidity.

Drosophila construction. The flies were driven via the muscular driver MHC-GAL4. MHC-GAL4 virgin flies were crossed with W1118 male flies, and the F1 generation genes were W1118/+; MHC-GAL4/+, which served as the control group. In the $P I N K 1^{B 9}$ disease group, UAS-PINK $1^{B 9} / \mathrm{FM} 7$;
MHC-GAL4 virgin flies were crossed with W1118 male flies, which produced the F1 generation with a genotype of UAS-PINK $1^{B 9} /+$; MHC-GAL4/+. In the overexpression (OE) intervention group, UAS-PINK $1^{B 9} / \mathrm{FM} 7$; MHC-GAL4 virgin flies were hybridized with male flies of UAS-Wnt2OE, and the F1 generation genotype was UAS-PINK $1^{B 9} / \mathrm{y}$; MHC-GAL4/Wnt2 $\mathrm{OE}, \mathrm{UAS}-P I N K 1^{B 9} / \mathrm{y}$. In the RNA interference (RNAi) intervention group, UAS-PINK1 ${ }^{B 9} /$ FM7; MHC-GAL4 virgin flies were crossed with males of UAS-Wnt2 RNAi Drosophila, and the F1 generation obtained had the genotype UAS-PINK $1^{B 9} / \mathrm{y}$; MHC-GAL4/Wnt2RNAi.

Morphological observation of Drosophila (24). Flies carrying the MHC-GAL4/UAS systems were grouped as follows: Normal control group (W1118), disease control group $\left(P I N K 1^{B 9}\right), W n t 2 \mathrm{OE}$ intervention group (PINK $1^{B 9} ; W_{n t 2 \mathrm{OE})}$ and Wnt 2 knockdown intervention group (PINK $1^{B 9}$; Wnt2 RNAi). On day 5, 100 male flies were selected from each group. After being anesthetized by $\mathrm{CO}_{2}$, the 100 flies were divided into transparent glass tubes with five flies per tube. After the flies completely woke up (after $\sim 1 \mathrm{~h}$ ), the shape of their wings and whether they could fly were observed. The number and the ratio of abnormal wings and flying were calculated. All assays were performed in triplicate and independently repeated three times.

Drosophila mRNA expression detection. The experimental groups were the same as aforementioned. On day 5, the head and abdomen were removed, and the chest was kept from 30 male flies from each group. TRIzol ${ }^{\circledR}$ reagent (Invitrogen; Thermo Fisher Scientific, Inc.) was used to extract the total RNA from the chest, according to the manufacturer's protocol. The primers were synthesized by Sangon Biotech Co., Ltd. and are presented in Table I. Subsequently, RNA samples were reverse transcribed into cDNA using PrimeScript ${ }^{\mathrm{TM}}$ reverse transcription (RT) reagent kit with gDNA Eraser (Takara Bio, Inc.). Following the manufacturer's instructions, this reaction was a two-step process. The first step was to remove the genomic DNA, adding $1 \mu \mathrm{l}$ gDNA eraser and $2 \mu \mathrm{l}$ gDNA eraser buffer to the RNA sample, which was then incubated at $42^{\circ} \mathrm{C}$ for $2 \mathrm{~min}$. The second step was to synthesize cDNA by adding the $1 \mu \mathrm{l}$ enzyme, $1 \mu \mathrm{l}$ primer, $4 \mu \mathrm{l}$ buffer and $4 \mu \mathrm{l}$ RNase-free $\mathrm{H}_{2} \mathrm{O}$ to the $10 \mu \mathrm{l}$ gDNA eraser-treated sample. The reaction temperature was $37^{\circ} \mathrm{C}$ for $15 \mathrm{~min}$ and $85^{\circ} \mathrm{C}$ for 5 sec. Quantitative PCR (qPCR) was conducted using an Applied Biosystems ABI 7500 system (Thermo Fisher Scientific, Inc.) using Power SYBR ${ }^{\circledR}$ Green PCR Master mix (Thermo Fisher Scientific, Inc.). The RT PCR conditions were as follows: Initial denaturation at $50^{\circ} \mathrm{C}$ for $20 \mathrm{sec}$ and $95^{\circ} \mathrm{C}$ for $10 \mathrm{~min}$, followed by 40 cycles of $95^{\circ} \mathrm{C}$ for $15 \mathrm{sec}$; annealing at $60^{\circ} \mathrm{C}$ for $1 \mathrm{~min}$ and extension at $72^{\circ} \mathrm{C}$ for $1 \mathrm{~min}$. Moreover, $18 \mathrm{~S}$ served as an endogenous control for data normalization. The $2^{-\Delta \Delta \mathrm{Cq}}$ method (25) was used to analyze the relative expression.

Western blotting for detection of protein expression. Drosophila grouping was the same as aforementioned. The the chest tissue of 20 Drosophila was cut on ice. Fresh tissues were lysed with $200 \mu 1$ ice-cold RIPA buffer (Solarbio Science \& Technology Co., Ltd.) containing $1 \mathrm{mM}$ phenylmethylsulfonyl 
Table I. Primer sequences used in the reverse transcription-quantitative PCR assay.
Gene
Primer sequence $\left(5^{\prime} \rightarrow 3^{\prime}\right)$

$18 S$

Forward: TCTAGCAATATGAGATTGAGCAATAAG Reverse: AATACACGTTGATACTTTCATTGTAGC

ND1 Forward: GTTATAGTAGCTGGTTGGTCGTC Reverse: AAGGAGTCCGATTAGTTTCAGC

ND42 Forward: CAAGAAGATGCTCGACTGGC Reverse: TGTCTGCATTGTAGCCAGGA

ND75 Forward: AAGCTCTTCCTTACCGAACTG Reverse: ATCGATGCTGCTCACCTTAC

$s d h B \quad$ Forward: CCATCGCCGAGATCAAGAAG Reverse: GGGACGAACTGGGAGTAGA

$c y t b$ Forward: GGATACGTATTACCTTGAGGACAAA Reverse: CAACAGCAAATCCACCTCATAATC

COX1 Forward: TGGTGGATTTGGAAATTGATTAGTG Reverse: GTAAAGAAAGAGCAGGAGGTAGAA

$P G C-1 \alpha$ Forward: AAGACGTGCCTTCTGTCGTTCATC Reverse: ATTCGGTGCTGGTGCTTCCTTG

FOXO Forward: CTCATCCAATGCCAGTTCCT Reverse: TCATCGTTGTGTTCTGGTAGTC

$\mathrm{ND}$, NADH-ubiquinone oxidoreductase chain 1 ; sdhB, succinate dehydrogenase complex subunits B; cytb, Cytochrome b; COX1, Cyclooxygenase 1 ; PGC- $1 \alpha$, PPARG coactivator $1 \alpha$; FOXO, forkhead box sub-group $\mathrm{O}$.

fluoride (Beijing Solarbio Science \& Technology Co., Ltd.). Subsequently, tissues were ground to a homogenate, followed by centrifugation at $14,000 \mathrm{x}$ for $15 \mathrm{~min}$ at $4^{\circ} \mathrm{C}$. Then, $120 \mu \mathrm{l}$ supernatant liquid was collected and added to $40 \mu 14$ Xloading buffer (Beijing Solarbio Science \& Technology Co., Ltd.), mixed and boiled at $100^{\circ} \mathrm{C}$ for $10 \mathrm{~min}$. The samples were stored at $-20^{\circ} \mathrm{C}$ prior to further experiments.

For analytical SDS-PAGE, $10 \mu \mathrm{l}$ protein was loaded per lane to $5 \%$ concentrated gel $(30 \%$ acrylamide, $10 \%$ SDS; 10\% APS; 1 M Tris-HCl pH 6.8; TEMED) and dissociated in $10 \%$ separation gel (30\% acrylamide; $10 \%$ SDS; $10 \%$ APS; 1.5 $\mathrm{M}$ Tris- $\mathrm{HCl} \mathrm{pH}$ 8.8; TEMED). The proteins were then transferred to 0.2- $\mu \mathrm{m}$ PVDF membranes (Beijing Solarbio Science and Technology Co., Ltd.), blocked in 5\% milk for $2 \mathrm{~h}$ at room temperature and washed in TBS-T (TBS, $3 \mathrm{M} \mathrm{NaCl}$, 200M Tris; pH 7.5, containing $0.1 \%$ Tween-20). Subsequently, overnight incubation at $4^{\circ} \mathrm{C}$ was performed with the following primary antibodies: Rabbit anti-MnSOD (1:1,000; cat. no. ab13534; Abcam; polyclonal), rabbit anti- $\alpha$ tubulin (1:1,000; cat. no. ab52866; Abcam; monoclonal) and mouse anti- $\beta$-catenin $(1: 1,000$; cat. no. AB_528089; Developmental Studies Hybridoma Bank; monoclonal). All the primary antibodies were Drosophila-specific. Following washing with TBS-T, the membrane was incubated with the corresponding secondary antibody, horseradish peroxidase-AffiniPure Goat Anti-Rabbit/Mouse IgG (H+L; 1:5,000; cat. nos. EM35111-01 and EM35110-01; Emarbio Science and Technology Co., Ltd.), for $1 \mathrm{~h}$ at room temperature. All the resulting immune complexes were visualized with chemiluminescence reagent (Thermo Fisher Scientific, Inc.,), followed by imaging using Image Lab 5.1 (National Institutes of Health). The target protein bands were quantified by scanning densitometry using ImageJ software (v.1.49v; National Institutes of Health).

ATP, malondialdehyde (MDA) and reactive oxygen species (ROS). In total, ten Drosophila thoraxes were cut on ice from each group, and 1,000 $\mu$ l lysate was added to homogenize the chest tissue on ice. Samples were then heated at $100^{\circ} \mathrm{C}$ for $2 \mathrm{~min}$ and centrifuged at $12,000 \mathrm{x}$ g for $5 \mathrm{~min}$ at $4^{\circ} \mathrm{C}$. The thoracic ATP level was measured using a luciferase-based bioluminescence assay (cat. no. S0027; Beyotime Institute of Biotechnology). Then, 40 fly chests from each group were ground by adding $500 \mu \mathrm{l} 1 \mathrm{X}$ PBS and centrifuge at $1,425 \mathrm{x} \mathrm{g}$ for $10 \mathrm{~min}$ at $4^{\circ} \mathrm{C}$. The supernatant was collected and the MDA content was measured by the thiobarbituric acid method (26). Analyses were performed according to the instructions of the reagent kit for MDA (cat. no. A003-1-2; Nanjing Jiancheng Bioengineering Institute). ROS were measured using the CellROX Orange reagent (cat. no. BB-470512; Shanghai Bio-Tech Co., Ltd.). The thoraxes of 30 Drosophila were obtained, homogenized, centrifuged at 1,000 x g for $10 \mathrm{~min}$ at $4^{\circ} \mathrm{C}$ and the supernatant was collected. The supernatant and $20 \mu \mathrm{M}$ CellROX Orange Reagent were mixed and incubated at $37^{\circ} \mathrm{C}$ in the dark for $30 \mathrm{~min}$, and the fluorescence intensity at 510 and $610 \mathrm{~nm}$ (maximum excitation light and maximum emission wavelength) was measured using a multi-function microplate reader.

Transmission electron microscopy analysis. Drosophila was grouped as aforementioned, and 10 male flies from each group F1 generation were randomly selected on day 5. Flies were anesthetized by $\mathrm{CO}_{2}$, and the chest was cut carefully so as not to damage the muscle tissue. Thoraxes were fixed overnight at $4^{\circ} \mathrm{C}$ in $2.5 \%$ glutaraldehyde, washed several times with $0.1 \mathrm{~mol} / 1$ phosphate buffer and post-fixed in $1 \%$ osmiumtetroxide in distilled water for $2 \mathrm{~h}$ at room temperature. The samples were dehydrated in a 50,70 and 90\% graded ethanol series, and embedded in Epoxy resin for $48 \mathrm{~h}$ at room temperature. The polymerization conditions in the polymerization tank were $36^{\circ} \mathrm{C}$ for $24 \mathrm{~h}, 45^{\circ} \mathrm{C}$ for $12 \mathrm{~h}$ and $65^{\circ} \mathrm{C}$ for $48 \mathrm{~h}$.

The embedded polymer samples were cut into $1 \mu \mathrm{m}$ sections using a Leica UC7 ultrathin slicer, stained with $1 \%$ toluidine blue for $30 \mathrm{sec}$ at room temperature and observed under an optical microscope (magnification, x100). The samples were then cut into ultra-thin sheets $(70 \mathrm{~nm})$, stained with $3 \%$ uranyl acetate for $15 \mathrm{~min}$ at room temperature and $3 \%$ lead citrate for $15 \mathrm{~min}$ at room temperature. Sections were observed with a Hitachi H-7650 transmission electron microscope (magnification, $\mathrm{x} 30,000)$.

Statistical analysis. Statistical analysis of data was performed using SPSS 16.0 (IBM Corp.). Normally distribution continuous variable data were compared by one-way ANOVA followed by Bonferroni's post hoc test. Data are presented as the mean $\pm \mathrm{SD}$. $\mathrm{P}<0.05$ was considered to indicate a statistically significant difference. 
Aa

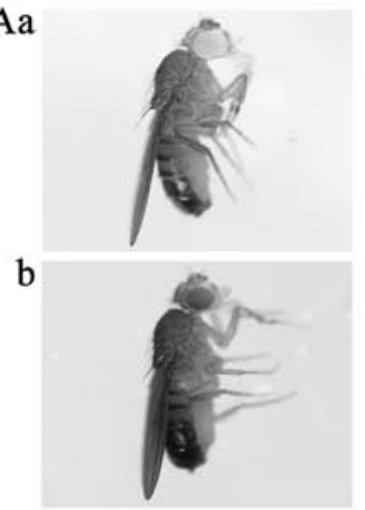

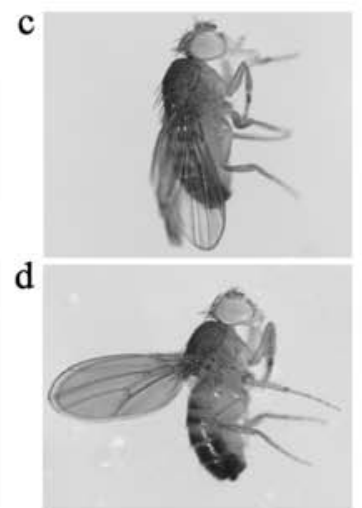

B

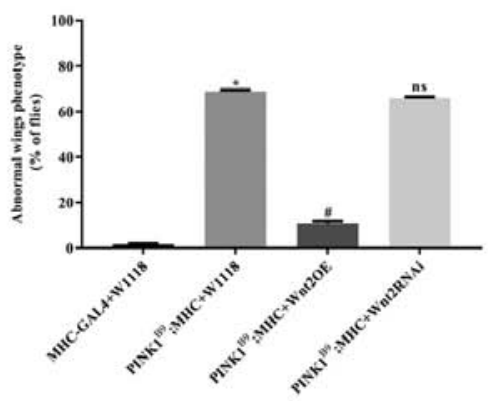

$\mathrm{C}$

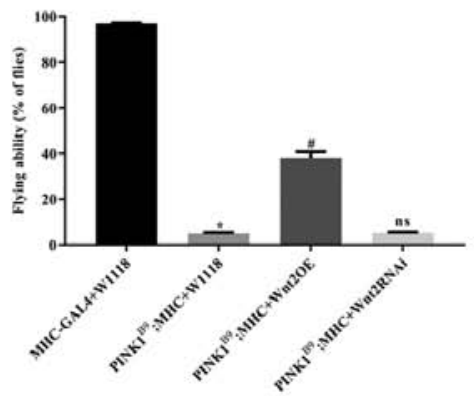

Figure 1. Wnt 2 overexpression suppresses PINK $1^{B 9}$ mutant phenotypes. (A) Side view of Drosophila wings. (Aa) Control flies, (Ab) PINK1 ${ }^{B 9}$ flies, (Ac) Wnt2OE flies and (Ad) Wnt2RNAi flies. Both (B) wing phenotype and (C) flying ability were abnormal in $P I N K l^{B 9}$ flies. Abnormal wing phenotype and flying rate was restored in Wnt $2 \mathrm{OE}$ flies compared with in $P I N K 1^{B 9}$ flies. $\mathrm{n}=20,5$-day-old males. ${ }^{*} \mathrm{P}<0.05$ vs. the control flies. ${ }^{"} \mathrm{P}<0.05$ vs. the PINKI $^{B 9}$ flies. ns vs. the $P I N K 1^{B 9}$ flies. PINK1, PTEN induced putative kinase 1; Wnt2OE, Wnt2 overexpression; Wnt2RNAi, Wnt2 RNA interference; ns, not significant.

Aa

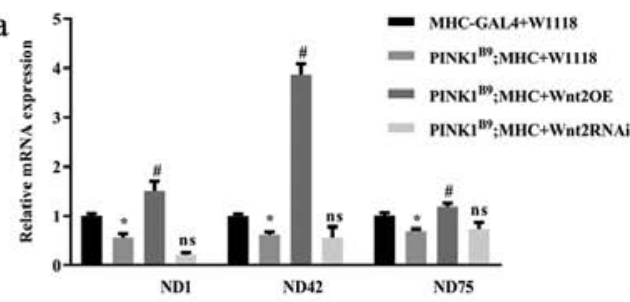

B

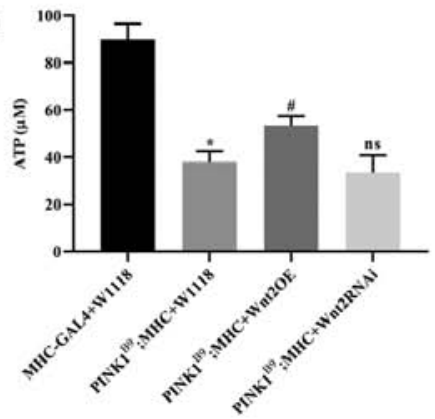

b

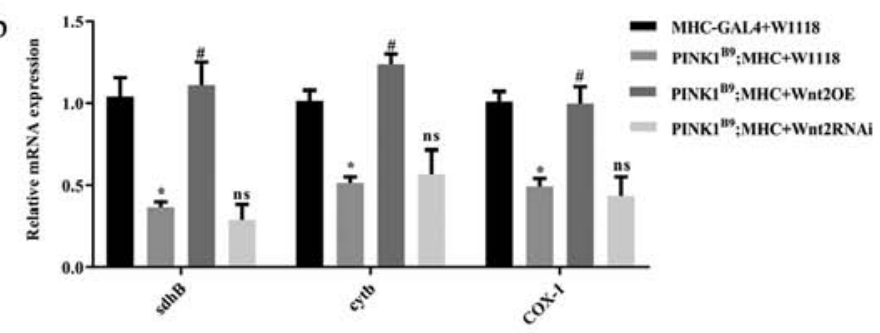

$\mathrm{Ca}$
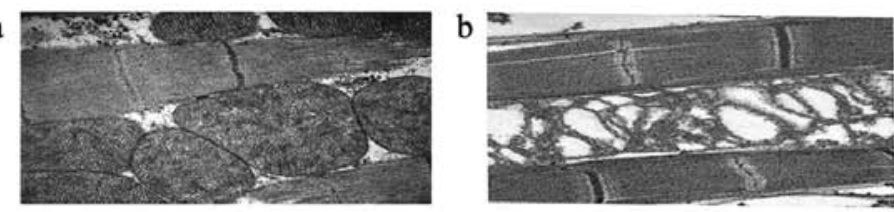

c

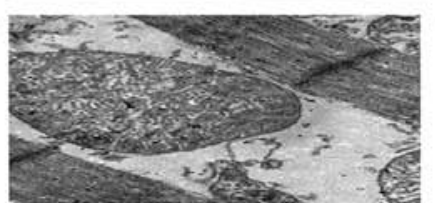

$\mathrm{d}$

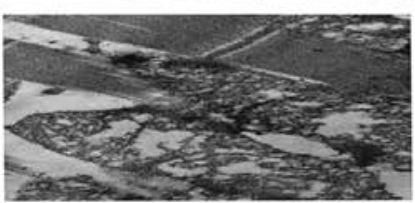

Figure 2. Overexpression of Wnt2 rescues mitochondrial function. (A) Overexpression of Wnt2 increased mitochondrial complex subunit-related genes. (Aa) mRNA level of mitochondrial complex subunit ND1, ND42 and ND75, (Ab) mRNA level of mitochondrial complex subunit sdhB, cytb and COX-1. (B) ATP levels in flies. (C) Abnormal mitochondrial morphology of $P I N K 1^{B 9}$ flies was restored by Wnt2 overexpression. (Ca) Control flies, (Cb) PINK1B9 flies, (Cc) Wnt2OE flies and (Cd) Wnt2RNAi flies. Magnification, x30,000. Scale bar, $1 \mu \mathrm{m}$. $\mathrm{n}=30$, 5-day-old males. ${ }^{*} \mathrm{P}<0.05$ vs. the control flies. ${ }^{\# P}<0.05$ vs. the $P I N K 1^{B 9}$ flies. ns vs. the $P I N K 1^{B 9}$ flies. PINK1, PTEN induced putative kinase 1; Wnt2OE, Wnt2 overexpression; Wnt2RNAi, Wnt2 RNA interference; ns, not significant; ND, NADH-ubiquinone oxidoreductase chain 1; sdhB, succinate dehydrogenase complex subunits B; cytb, Cytochrome b; COX1, Cyclooxygenase 1 .

\section{Results}

Wht 2 overexpression rescues the abnormal phenotype caused by $P I N K 1^{B 9}$ mutation. The Wnt 2 gene was specifically expressed in Drosophila flight muscles using the MHC-GAL4 promoter. The phenotypic changes of Drosophila were demonstrated by changes in wing morphology and flight ability. In the normal control group, most of the wings completely overlapped and were parallel to the body (Fig. 1A). However, in the transgenic disease model of $P I N K 1^{B 9}$ the wings had bifurcations, erections and sagged. Furthermore, compared with the low rate of abnormal wings in the control group, the disease group had a significantly higher rate of abnormal wings (Fig. 1B) and a significant decrease in flight ability (Fig. 1C). In the Wnt2OE intervention group, the incidence of wing anomalies was significantly reduced and the flight capabilities were improved, compared with the disease group. Moreover, there were no significant differences between the Wnt2 RNAi intervention group and the PD disease model group. Therefore, the present results suggested that $W n t 2 \mathrm{OE}$ may have a protective effect on the phenotype of $P I N K 1^{B 9}$ transgenic Drosophila.

Wnt2 gene overexpression enhances mitochondrial function in PINK $1^{B 9}$ transgenic Drosophila. RT-qPCR results demonstrated that in $P I N K 1^{\mathrm{B} 9}$ disease model group, the mRNA expression levels of the mitochondrial complex subunit-related genes, Complex I [NADH-ubiquinone oxidoreductase chain 1 (ND1), ND42 and ND75], Complex II (succinate dehydro- 
A

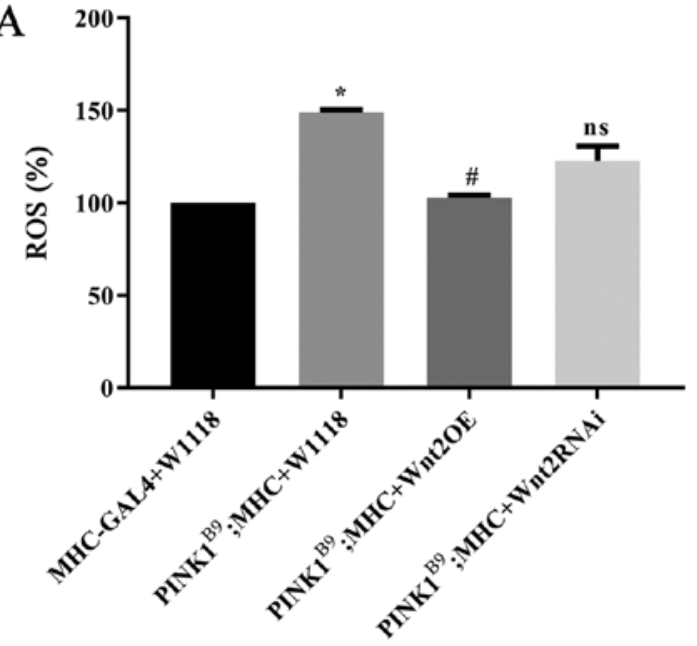

$\mathrm{Ca}$

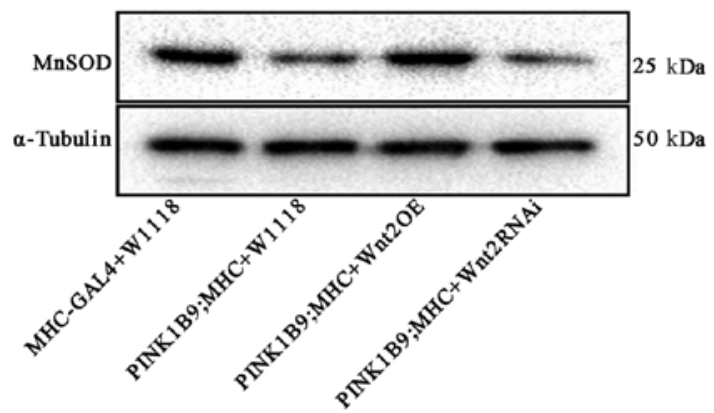

B
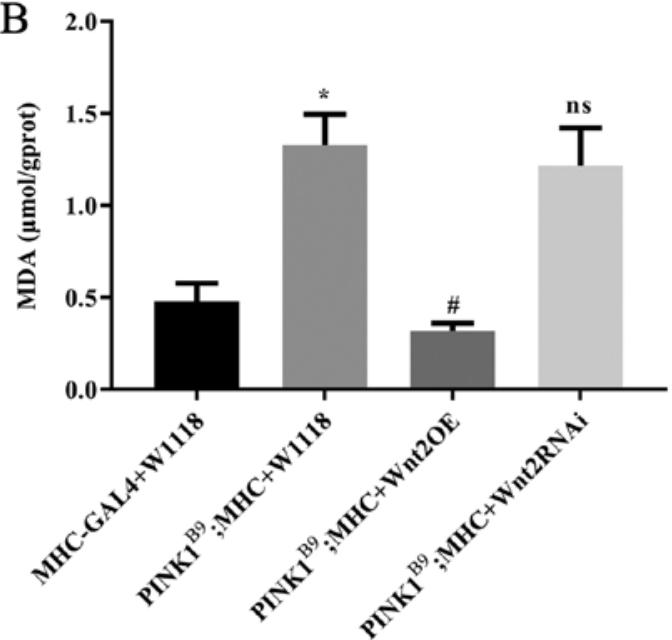

b

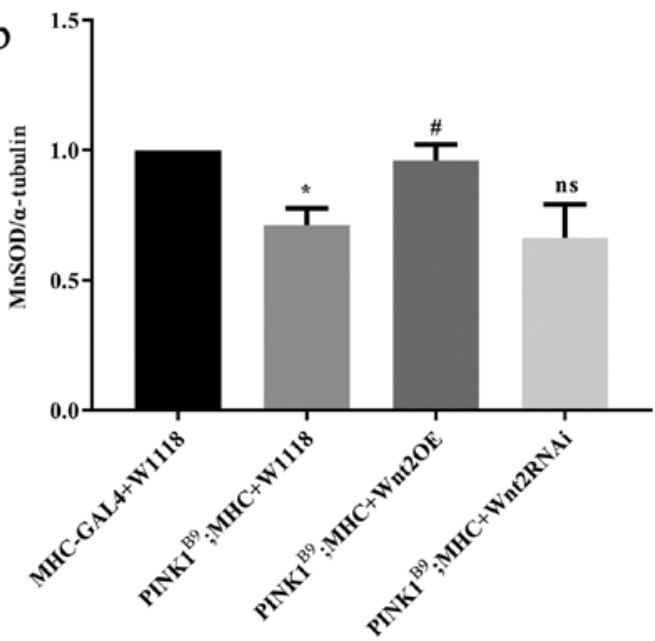

Figure 3. Wnt2 overexpression alleviates oxidative stress in $P I N K 1^{B 9}$ flies. (A) ROS levels. (B) MDA levels. (C) Protein expression levels of MnSOD were quantified by western blotting. (Ca) Western blot analysis of MnSOD protein, (Cb) Relative quantitative analysis of MnSOD protein. $\mathrm{n}=40$, 5-day-old males. ${ }^{*} \mathrm{P}<0.05$ vs. the control flies. ${ }^{\prime \prime} \mathrm{P}<0.05$ vs. the $P I N K 1^{B 9}$ flies. ns vs. the $P I N K{ }^{B 9}$ flies. ROS, reactive oxygen species; MDA, malondialdehyde; MnSOD, manganese superoxide dismutase; PINK1, PTEN induced putative kinase 1; Wnt2OE, Wnt2 overexpression; Wnt2RNAi, Wnt2 RNA interference; ns, not significant.

genase complex subunits B), Complex III (Cytochrome b) and Complex IV (Cyclooxygenase 1), decreased significantly. While Wnt2OE intervention in $P I N K 1^{\mathrm{B} 9}$ transgenic Drosophila increased the mRNA expression levels of these related genes $(\mathrm{P}<0.05)$, in the Wnt2 RNAi intervention group there was no significant difference compared with the disease model group (Fig. 2A). The results of the ATP assay demonstrated that ATP production in the PD model group was decreased. Furthermore, the amount of ATP produced by mitochondria in the Wnt2OE intervention group was $\sim 1.5$ times higher compared with the disease model group (Fig. 2B). Ultrastructural transmission electron microscopy analysis identified that mitochondria were disrupted in $P I N K 1^{B 9}$ transgenic Drosophila, and mitochondrial morphology was not recognizable. Moreover, Wnt2OE could rescue mitochondrial defects in $P I N K 1^{B 9}$ flies (Fig. 2C). Therefore, the present results suggested that overexpression of Wnt 2 can improve the mitochondrial function of $P I N K 1^{B 9}$ transgenic Drosophila.

Wnt2OE reduces oxidative stress damage in $P I N K 1^{B 9}$ transgenic Drosophila. ROS production in the PINK $1^{B 9}$ disease model group was significantly higher compared with the normal control group $(\mathrm{P}<0.05 ;$ Fig. 3A). Furthermore, following Wnt2OE intervention in $P I N K^{B 9}$ transgenic Drosophila, ROS production was significantly reduced $(\mathrm{P}<0.05)$ and almost returned to normal levels. MDA, a commonly used indicator of lipid peroxidation injury (27), was significantly increased in the $P I N K 1^{B 9}$ disease model group compared with the normal control $(\mathrm{P}<0.05$; Fig. 3B). Moreover, after Wnt $2 \mathrm{OE}$ intervention, MDA production was reduced $(\mathrm{P}<0.05)$. It was demonstrated that the content of ROS and MDA were not significantly different between the Wnt2RNAi intervention groups and the disease model group (Fig. 3A and B).

Western blot analysis results revealed that the protein expression of MnSOD in the PINK $1^{\mathrm{B} 9}$ disease model group was significantly lower compared with the normal control group $(\mathrm{P}<0.05$; Fig. $3 \mathrm{C})$. However, the expression of MnSOD was significantly increased $(\mathrm{P}<0.05)$ following Wnt2OE intervention in the $P I N K 1^{B 9}$ disease model. Collectively, the present results indicated that $W n t 2$ overexpression reduced oxidative damage in $P I N K 1^{B 9}$ transgenic Drosophila.

Possible mechanism of Wnt2 overexpression-mediated protection. Western blot analysis results revealed that there was no 

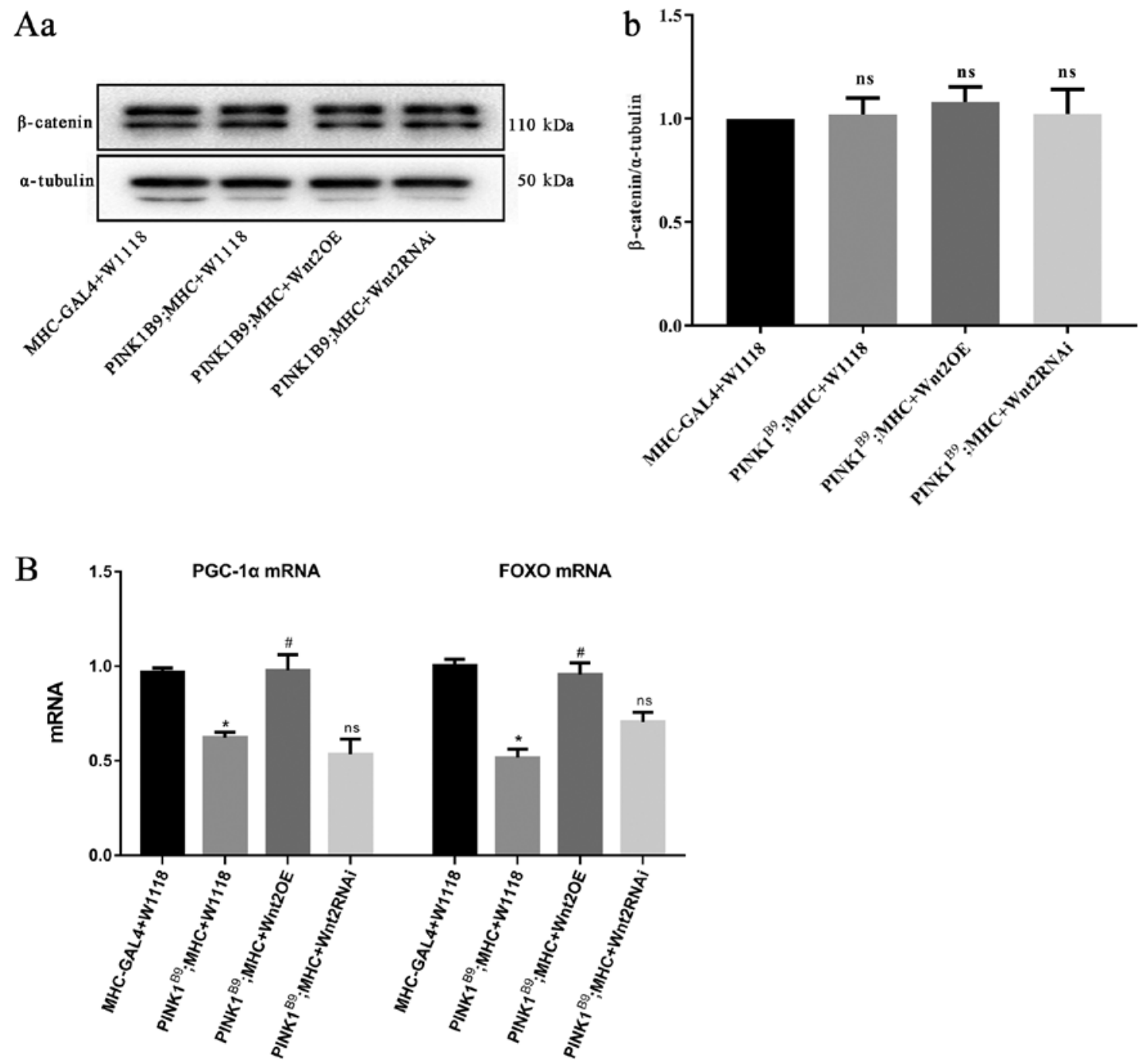

Figure 4. Wnt 2 overexpression activates the FOXO/PGC-1 $\alpha$ pathway to protect $P I N K l^{B 9}$ flies. (A) Protein expression of $\beta$-catenin was quantified by western blotting. (Aa) Western blot analysis of $\beta$-catenin protein, (Ab) Relative quantitative analysis of $\beta$-catenin protein. (B) mRNA expression levels of PGC-1 $\alpha$ and FOXO were detected by reverse transcription-quantitative PCR. $\mathrm{n}=30,5$-day-old males. ${ }^{*} \mathrm{P}<0.05$ vs. the control flies. ${ }^{\prime \prime} \mathrm{P}<0.05$ vs. the $P I N K 1^{B 9}$ flies. ns vs. the $P I N K 1^{B 9}$ flies. PINK1, PTEN induced putative kinase 1; Wnt2OE, Wnt2 overexpression; Wnt2RNAi, Wnt2 RNA interference; PGC-1 $\alpha$, PPARG coactivator $1 \alpha$; FOXO, forkhead box sub-group O; ns, not significant.

significant difference in the protein expression of $\beta$-catenin, a key molecule of the classic Wnt $/ \beta$-catenin signaling pathway (28), between each group (Fig. 4A). Moreover, RT-qPCR showed that the mRNA expression levels of $F O X O$ and $P G C$ - $1 \alpha$ were decreased in the $P I N K 1^{B 9}$ disease model group, and were increased following Wnt2OE intervention in the $P I N K 1^{B 9}$ disease model (Fig. 4B). Therefore, it can be speculated that Wnt2 may activate the FOXO/PGC-1 $\alpha$ signaling pathway to regulate mitochondrial function and inhibit anti-oxidative stress-induced damage.

\section{Discussion}

In Drosophila melanogaster, mutations in PINK1 causes phenotypic abnormalities and decrease exercise capacity, resulting in dysfunction of mitochondria and decreased ATP levels, which leads to selective degeneration of DA neurons and sensitivity to stress $(16,17)$. In the present study, the disease model demonstrated a pathological phenotype that was consistent with previous experimental results $(24,29)$, including a high abnormal wing rate, low flight rate, lower mRNA expression of mitochondrial complex subunits and lower ATP level, which suggested that PINK1 mutations could cause serious damage in mitochondria. Furthermore, overexpression of Wnt2 gene rescued the phenotype of PINKI ${ }^{B 9}$ transgenic Drosophila, increased ATP production level and enhanced the expression of the mitochondrial biosynthesis-related gene $P G C-1 \alpha$. PGC- $1 \alpha$ is a transcriptional cofactor for numerous mitochondrial proteins, which can regulate mitochondrial function to meet cellular needs and protect from oxidative damage (30). Therefore, it is speculated that Wnt2OE inhibited mitochondrial dysfunction caused by the PINKI mutation and eventually protects $P I N K I^{B 9}$ transgenic fruit flies.

When mitochondria are damaged, particularly damage to the mitochondrial respiratory chain complex, electron chain leakage may occur and cause the production of superoxide and hydrogen peroxide (31). These products, not only participate in the damage of DNA, proteins and lipids, but also pose a serious threat to cells and tissues (31). Mitochondrial dysfunction and oxidative stress play key roles in the development of 
PD, as both lead to excessive ROS production (32). This in turn leads to damage and death of DA neurons (33). A small level of ROS is essential for normal physiological functions; however, the accumulation of large amounts of ROS further destroys mitochondria and exacerbates oxidative stress (34). It has been shown that maintenance of ATP and inhibition of ROS production protects DA neurons (35). Reactive oxygen damages polyunsaturated lipids and forms MDA, which is a marker of lipid damage in oxidative stress (36). Furthermore, the present results indicated that the PINK1 mutation increased ROS and MDA levels, but reduced MnSOD protein expression.

MnSOD, which is mainly distributed in the mitochondrial matrix, is an important scavenger for the superoxide anion that is produced during mitochondrial oxidative phosphorylation (37). Previous findings have shown that overexpression of $M n S O D$ serves an important role in the protection of PINK1-mutant PD Drosophila (38). In the PINK1 mutants, the present study identified increased ROS and MDA production, and a reduced MnSOD, thus suggesting that the PINKI mutation leads to oxidative stress damage. Moreover, overexpression of Wnt2 improved mitochondrial function, which also partially reduced ROS production. However, Wnt2 overexpression also appeared to directly participate in the regulation of oxidative stress levels in $P I N K 1^{B 9}$ transgenic Drosophila, as it not only reduced ROS and MDA production levels, but also increased the protein expression levels of MnSOD.

The Drosophila gene Wnt2, homologous to the human gene $W n t 7 a$, is involved in the $\mathrm{Wnt} / \beta$-catenin signaling pathway (39). However, it remains unknown whether the protective effect of Wnt2 overexpression on PINK $1^{B 9}$ transgenic Drosophila is related to the $\mathrm{Wnt} / \beta$-catenin signaling pathway. The present results indicated that $W n t 2$ overexpression did not increase the protein expression of $\beta$-catenin, a key protein of the Wnt/ $\beta$-catenin signaling pathway (28), which suggested that it did not protect $P I N K 1^{B 9}$ transgenic flies via the Wnt $/ \beta$-catenin signaling pathway. Furthermore, a previous study found that $W n t 2$ does not act via the $\mathrm{Wnt} / \beta$-catenin pathway, but activates the non-canonical pathway (40). Thus, this raises the question of how Wnt 2 improves mitochondrial function, reduces oxidative damage and enhances antioxidant capacity. The present study evaluated the expression of FOXO, a gene associated with mitochondrial oxidative stress, which regulates the expression levels of $P G C-1 \alpha$ and $M n S O D(38,41)$. Under conditions of mitochondrial dysfunction and oxidative stress caused by PINK1 mutation, the present study hypothesized that the overexpression of Wnt 2 directly regulates the expression of PGC- $1 \alpha / \mathrm{FOXO} / \mathrm{MnSOD}$, improves mitochondrial function and improves antioxidant capacity to rescue $P I N K 1^{B 9}$ transgenic fruit flies.

The FOXO family members are key regulators of neuronal processes, such as dendritic structural function and memory consolidation $(42,43)$. FOXO is also an important regulator of cellular stress response, which enhances cellular antioxidant defenses (44). A previous study revealed that the expression of genes, such as $M n S O D$, could be controlled by the forkhead transcription factor $F O X O 3 a$ (44). Moreover, $P G C$ - $1 \alpha$ has been shown to regulate $F O X O$ activity in different systems. For example, it has been reported that $P G C-1 \alpha$ is a positive regulator of fasting-induced hepatic gluconeogenesis, which is mediated by its interaction with FOXOla (45). Similarly, overexpression of $P G C$ - $1 \alpha$ enhances the stimulatory effect of FOXOla on selenoprotein $\mathrm{P}$ promoter activity and insulin attenuation (46). $P G C-1 \alpha$ has also been shown to interact with $\mathrm{FOXO3a}$, which regulates antioxidant gene expression in endothelial cells and skeletal muscle (47). In addition, upregulation of $P G C-1 \alpha$ and $F O X O 3 a$ protects against oxidative stress injury induced by a high-fat diet and inhibits adipocyte apoptosis $(47,48)$. The Wnt signaling pathway is also involved in the regulation of mitochondrial energy metabolism and oxidative stress (49). When ROS levels exceed the body's ability to scavenge, Wnt and $\beta$-catenin interact with $F O X O$ under stimuli of oxidative stress $(13,50)$. Furthermore, FOXO1 interacts with $P G C-1 \alpha$ in different systems $(45,46)$. PGC- $1 \alpha$ is a mitochondrial energy-metabolizing enzyme. When ROS are in excess, the human body can enhance the detoxification ability of mitochondrial ROS by increasing the expression levels of $F O X O 1$ and $P G C-1 \alpha$ to promote the expression of downstream antioxidant systems, such as $M n S O D$ (49).

To the best of our knowledge, the condition of mitochondrial dysfunction caused by PINK1 mutation and oxidative stress remains to be determined. Although the Wnt signaling pathway is linked to the FOXO signaling pathway via $\beta$-catenin (50), the present results suggested that Wnt 2 did not change the $\beta$-catenin protein expression level, but did affect the mRNA expression levels of PGC-1 $\alpha$ and FOXO, and the expression levels of their target protein MnSOD. Based on these results, it can be speculated that Wnt2 may be directly involved in the PGC- $1 \alpha / \mathrm{FOXO} / \mathrm{MnSOD}$ signaling pathway; however, the specific mechanism remains to be investigated. Moreover, the regulation of signaling pathways is complex and there will be cross-effects between the pathways (47-48), with both upregulation and inhibition of the expression of related factors leading to cascade reactions. Therefore, it will be beneficial to examine how the Wnt2 pathway interacts with or influences the PGC- $1 \alpha / \mathrm{FOXO} / \mathrm{MnSOD}$ pathway in future experiments.

The present study demonstrated that Wnt 2 overexpression protected $P I N K 1^{B 9}$ transgenic flies by improving flight muscle and mitochondrial morphology, and enhancing mitochondrial complex I and II function. Furthermore, it was found that Wnt 2 overexpression exhibited a protective effect on early mitochondria and oxidative stress-related PD by improving mitochondrial function and reducing oxidative stress damage. However, the present study does have some limitations. First, the model is monotonous and limited to fruit flies, and thus requires further examination in higher animal models such as mice. Secondly, further research into the underlying pathways and mechanisms is required, such as whether it is the experimental effects that are related to the non-classical Wnt signaling pathway. Furthermore, how Wnt2 cross-links with the PGC-1 $\alpha / \mathrm{FOXO} / \mathrm{MnSOD}$ signaling pathway is not fully understood. In the PINK1 mutant PD Drosophila model of mutation constructed by Park et al (24), mitochondrial dysfunction and oxidative stress injury are primarily exhibited in the early stage, while DA neuronal loss predominantly occurs in the middle and late stages of the Drosophila, which is after 25 days $(24,50)$. This is consistent with the progressive DA neuronal loss observed in clinical patients with PD. 
Thus, this may indicate that damage to DA neurons in PD is the result of further neurological damage caused by these pathogenic factors.

To the best of our knowledge, there are currently no treatments that mitigate disease progression or prevent neurodegeneration in all neurodegenerative disease. Therefore, it is necessary to develop interventions that are effective prior to severe damage of the neuronal in the early stages of PD. The present results indicated that $W n t 2$ overexpression had a protective effect on early mitochondrial damage and oxidative stress in PD, improving mitochondrial function and reducing oxidative stress damage. Due to the limitations of the present study, the specific mechanism of action of Wnt2 is not fully understood. However, the improvement of mitochondrial function and the reduction of oxidative stress damage can support the experimental results of the Drosophila model, providing a strong basis for future experiments.

In conclusion, the present results suggested that the Wnt2 gene may have a protective effect on $P I N K 1^{B 9}$ transgenic Drosophila. Therefore, it can be hypothesized that the reduction of oxidative stress and the restoration of mitochondrial function via Wnt2 gene overexpression in the PINK1 mutant transgenic Drosophila may be related to the PGC-1 $\alpha / \mathrm{FOXO} / \mathrm{MnSOD}$ signaling pathway.

\section{Acknowledgements}

The authors would like to thank Dr Yu-Feng Yang, from Institute of life Sciences of Fuzhou University, for donating the PINK1 $1^{\mathrm{B} 9}$ Drosophila model; Dr Ru-Jia Liao, Dr Jing-Xin Mo and Dr Qing-Tuan Meng, from Guangxi Clinical Research Center for Neurological Disease of Affliated Hospital of Guilin Medical University, for sharing their expertise; Miss Liang-Xian Li, intermediate research assistant, from Guangxi Key Laboratory of Brain and Cognitive Neuroscience of Guilin Medical University; Miss Ying Cui and Miss Xiao-Jun Diao, PhD candidate, Department of Neurology from Xiangya School of Medicine of Central South University; Miss Wen-Jing Wang, postgraduate student, Department of Neurology from Guilin Medical University; Miss Fang Shi (intermediate research assistant), Miss Ning Tian (inter- mediate research assistant) and Miss Mei-Rong Chen (intermediate research assistant), from Guangxi Clinical Research Center for Neurological Disease of Affliated Hospital of Guilin Medical University, for their help.

\section{Funding}

The present study was supported by the National Natural Science Foundation of China (grant nos. 81460180 and 31460256), 2017 Youth and Middle School Teachers' Basic Ability Improvement Project of the Education Department of Guangxi Zhuang Autonomous Region (grant no. 30606017018) and the Innovation Project of Guangxi Graduate Education (grant no. YCSW2018206).

\section{Availability of data and materials}

The datasets used and/or analyzed during the present study are available from the corresponding author on reasonable request.

\section{Authors' contributions}

SRX was responsible for construction of the Drosophila models, western blotting, MDA and ROS level determination, and data analysis. XYW was responsible for construction of the Drosophila models, morphological observation of Drosophila, $\mathrm{O} 2 \mathrm{~K}$ detection and data statistics. SRX and XYW wrote the first draft of this article. XLF was responsible for electron microscopy analysis. XRC performed the ATP determination experiment. ZWW was responsible for mRNA expression level detection. QHL and LS undertook project funding, project design, manuscript revision and quality control. All authors read and approved the final manuscript.

\section{Ethics approval and consent to participate}

Not applicable.

\section{Patient consent for publication}

Not applicable.

\section{Competing interests}

The authors declare that they have no competing interests.

\section{References}

1. Zhou C, Huang Y, Shao Y, May J, Prou D, Perier C, Dauer W, Schon EA and Przedborski S: The kinase domain of mitochondrial PINK1 faces the cytoplasm. Proc Natl Acad Sci USA 105: 12022-12027, 2008.

2. Hatano Y, Li Y, Sato K, Asakawa S, Yamamura Y, Tomiyama H, Yoshino H, Asahina M, Kobayashi S, Hassin-Baer S, et al: Novel PINK1 mutations in early-onset parkinsonism. Ann Neurol 56: 424-427, 2004.

3. Liu S, Sawada T, Lee S, Yu W, Silverio G, Alapatt P, Millan I, Shen A, Saxton W, Kanao T, et al: Parkinson's disease-associated kinase PINK1 regulates Miro protein level and axonal transport of mitochondria. PLoS Genet 8: e1002537, 2012.

4. Wang W, Wang X, Fujioka H, Hoppel C, Whone AL, Caldwell MA, Cullen PJ, Liu J and Zhu X: Parkinson's disease-associated mutant VPS35 causes mitochondrial dysfunction by recycling DLP1 complexes. Nat Med 22: 54-63, 2016.

5. Wu Z, Wang Y, Lim J, Liu B, Li Y, Vartak R, Stankiewicz T, Montgomery S and Lu B: Ubiquitination of ABCE1 by NOT4 in response to mitochondrial damage links co-translational quality control to PINK1-directed mitophagy. Cell Metab 28: 130-144. e7, 2018.

6. Oh SE, Park HJ, He L, Skibiel C, Junn E and Mouradian MM: The Parkinson's disease gene product DJ-1 modulates miR-221 to promote neuronal survival against oxidative stress. Redox Biol 19: 62-73, 2018

7. Burbulla LF, Song P, Mazzulli JR, Zampese E, Wong YC, Jeon S, Santos DP, Blanz J, Obermaier CD, Strojny C, et al: Dopamine oxidation mediates mitochondrial and lysosomal dysfunction in Parkinson's disease. Science 357: 1255-1261, 2017.

8. Andersson ER, Saltó C, Villaescusa JC, Cajanek L, Yang S, Bryjova L, Nagy II, Vainio SJ, Ramirez C, Bryja V, et al: Wnt5a cooperates with canonical Wnts to generate midbrain dopaminergic neurons in vivo and in stem cells. Proc Natl Acad Sci USA 110: E602-E610, 2013.

9. Sousa KM, Villaescusa JC, Cajanek L, Ondr JK, Castelo-Branco G, Hofstra W, Bryja V, Palmberg C, Bergman T, Wainwright B, et al: Wnt2 regulates progenitor proliferation in the developing ventral midbrain. J Biol Chem 285: 7246-7253, 2010.

10. Marui T, Funatogawa I, Koishi S, Yamamoto K, Matsumoto H, Hashimoto O, Jinde S, Nishida H, Sugiyama T, Kasai K, et al: Association between autism and variants in the wingless-type MMTV integration site family member 2 ( WNT2) gene. Int J Neuropsychopharmacol 13: 443-449, 2010. 
11. Zhang X, Abreu JG, Yokota C, MacDonald BT, Singh S, Coburn KL, Cheong SM, Zhang MM, Ye QZ, Hang HC, et al: Tikil is required for head formation via Wnt cleavage-oxidation and inactivation. Cell 149: 1565-1577, 2012.

12. Kahn M: Can we safely target the WNT pathway? Nat Rev Drug Discov 13: 513-532, 2014

13. Parker JA, Vazquez-Manrique RP, Tourette C, Farina F, Offner N, Mukhopadhyay A, Orfila AM, Darbois A, Menet S, Tissenbaum HA, et al: Integration of $\beta$-catenin, sirtuin, and FOXO signaling protects from mutant huntingtin toxicity. $\mathrm{J}$ Neurosci 32: 12630-12640, 2012.

14. Arrázola MS, Ramos-Fernández E, Cisternas P, Ordenes D and Inestrosa NC: Wnt signaling prevents the $\mathrm{A} \beta$ oligomer-induced mitochondrial permeability transition pore opening preserving mitochondrial Structure in hippocampal neurons. PLoS One 12: e0168840, 2017.

15. Wei L, Ding L, Mo MS, Lei M, Zhang L, Chen K and Xu P: Wnt3a protects $\mathrm{SH}-\mathrm{SY} 5 \mathrm{Y}$ cells against 6-hydroxydopamine toxicity by restoration of mitochondria function. Transl Neurodegener 4: 11 , 2015.

16. Yang Y, Gehrke S, Imai Y, Huang Z, Ouyang Y, Wang JW, Yang L, Beal MF, Vogel H and Lu B: Mitochondrial pathology and muscle and dopaminergic neuron degeneration caused by inactivation of Drosophila Pink1 is rescued by Parkin. Proc Nat Acad Sci USA 103: 10793-10798, 2006.

17. Liu W, Acín-Peréz R, Geghman KD, Manfredi G, Lu B and Li C: Pink1 regulates the oxidative phosphorylation machinery via mitochondrial fission. Proc Natl Acad Sci USA 108 12920-12924, 2011.

18. Dung VM and Thao DTP: Parkinson's Disease Model. Adv Exp Med Biol 1076: 41-61, 2018.

19. Bilder D and Irvine KD: Taking stock of the Drosophila research ecosystem. Genetics 206: 1227-1236, 2017.

20. Barwell T, DeVeale B, Poirier L, Zheng J, Seroude F and Seroude L: Regulating the UAS/GAL4 system in adult Drosophila with Tet-off GAL80 transgenes. PeerJ 5: e4167, 2017.

21. Southall TD, Elliott DA and Brand AH: The GAL4 system: A versatile toolkit for gene expression in Drosophila. CSH Protoc: doi:10.1101/pdb.top49.

22. $\mathrm{Tu} \mathrm{H}$ and Casadaban MJ: The upstream activating sequence for L-leucine gene regulation in Saccharomyces cerevisiae. Nucleic Acids Res 18: 3923-3931, 1990

23. Robertson LK, Dey BK, Campos AR and Mahaffey JW: Expression of the drosophila gene disconnected using the UAS/GAL4 system. Genesis 34: 103-106, 2002.

24. Park J, Lee SB, Lee S, Kim Y, Song S, Kim S, Bae E, Kim J, Shong M and Kim JM: Mitochondrial dysfunction in Drosophila PINK 1 mutants is complemented by parkin. Nature 441: $1157-1161,2006$

25. Livak KJ and Schmittgen TD: Analysis of relative gene expression data using real-time quantitative PCR and the 2(-Delta Delta C(T)) Method. Methods 25: 402-408, 2001.

26. Rogério M, Cardoso $\mathrm{C}$ and Pestana C: Measurement of malondialdehyde in fish: A comparison study between HPLC methods and the traditional spectrophotometric test. Food Chem 112: 1038-1045, 2009.

27. Dragun Z, Filipović Marijić V, Krasnići N, Ramani S, Valić D, Rebok K, Kostov V, Jordanova M and Erk M: Malondialdehyde concentrations in the intestine and gills of Vardar chub (Squalius vardarensis Karaman) as indicator of lipid peroxidation. Environ Sci Pollut Res Int 24: 16917-16926, 2017.

28. Boonchai W, Walsh M, Cummings $M$ and Chenevix-Trench G: Expression of beta-catenin, a key mediator of the WNT signaling pathway, in basal cell carcinoma. Arch Dermatol 136: 937-938, 2000.

29. Gehrke S, Wu Z, Klinkenberg M, Sun Y, Auburger G, Guo S and Lu B: PINK1 and Parkin control localized translation of respiratory chain component mRNAs on mitochondria outer membrane. Cell Metabolism 21: 95-108, 2015.

30. Tsunemi T and La Spada AR: PGC-1 $\alpha$ at the intersection of bioenergetics regulation and neuron function: From Huntington's disease to Parkinson's disease and beyond. Prog Neurobiol 97: $142-151,2012$

31. Zorov DB, Juhaszova M and Sollott SJ: Mitochondrial reactive oxygen species (ROS) and ROS-induced ROS release. Physiol Rev 94: 909-950, 2014.
32. Ray A, Martinez BA, Berkowitz LA, Caldwell GA and Caldwell KA: Mitochondrial dysfunction, oxidative stress, and neurodegeneration elicited by a bacterial metabolite in a $\mathrm{C}$. elegans Parkinson's model. Cell Death Dis 5: e984, 2014.

33. Subramaniam SR and Chesselet MF: Mitochondrial dysfunction and oxidative stress in Parkinson's disease. Prog Neurobiol 106-107: $17-32,2013$

34. Kudryavtseva AV, Krasnov GS, Dmitriev AA, Alekseev BY, Kardymon OL, Sadritdinova AF, Fedorova MS, Pokrovsky AV, Melnikova NV, Kaprin AD, et al: Mitochondrial dysfunction and oxidative stress in aging and cancer. Oncotarget 7: 44879-44905, 2016.

35. Zuo L and Motherwell MS: The impact of reactive oxygen species and genetic mitochondrial mutations in Parkinson's disease. Gene 532: 18-23, 2013.

36. Weismann D, Hartvigsen K, Lauer N, Bennett KL, Scholl HP, Charbel Issa P, Cano M, Brandstätter H, Tsimikas S, Skerka C: Complement factor $\mathrm{H}$ binds malondialdehyde epitopes and protects from oxidative stress. Nature 478: 76-81, 2011.

37. Sheshadri P and Kumar A: Managing odds in stem cells: Insights into the role of mitochondrial antioxidant enzyme MnSOD. Free Radic Res 50: 570-584, 2016.

38. Koh H, Kim H, Kim MJ, Park J, Lee HJ and Chung J: Silent information regulator 2 (Sir2) and Forkhead box O (FOXO) complement mitochondrial dysfunction and dopaminergic neuron loss in Drosophila PTEN-induced kinase 1 (PINK1) null mutant. J Biol Chem 287: 12750-12758, 2012.

39. Swarup S and Verheyen EM: Wnt/Wingless signaling in Drosophila. Cold Spring Harb Perspect Biol 4: a007930, 2012.

40. Yu M, Ting DT, Stott SL, Wittner BS, Ozsolak F, Paul S, Ciciliano JC, Smas ME, Winokur D and Gilman AJ: RNA sequencing of pancreatic circulating tumour cells implicates WNT signalling in metastasis. Nature 487: 510-513, 2012.

41. Mukherjee S and Duttaroy A: Spargel/dPGC-1 is a new downstream effector in the insulin-TOR signaling pathway in Drosophila. Genetics 195: 433-441, 2013.

42. Salih DA, Rashid AJ, Colas D, de la Torre-Ubieta L, Zhu RP, Morgan AA, Santo EE, Ucar D, Devarajan K, Cole CJ, et al: FoxO6 regulates memory consolidation and synaptic function. Genes Dev 26: 2780-2801, 2012.

43. Sears JC and Broihier HT: FoxO regulates microtubule dynamics and polarity to promote dendrite branching in Drosophila sensory neurons. Dev Biol 418: 40-54, 2016.

44. Kops GJ, Dansen TB, Polderman PE, Saarloos I, Wirtz KW, Coffer PJ, Huang TT, Bos JL, Medema RH and Burgering BM: Forkhead transcription factor FOXO3a protects quiescent cells from oxidative stress. Nature 419: 316-321, 2002.

45. Puigserver P, Rhee J, Donovan J, Walkey CJ, Yoon JC, Oriente F, Kitamura Y,Altomonte J,Dong H,AcciliD, etal:Insulin-regulated hepatic gluconeogenesis through FOXO1-PGC-1alpha interaction. Nature 423: 550-555, 2003.

46. Speckmann B, Walter PL, Alili L, Reinehr R, Sies H, Klotz LO and Steinbrenner H: Selenoprotein P expression is controlled through interaction of the coactivator PGC-1alpha with FoxOla and hepatocyte nuclear factor 4alpha transcription factors. Hepatology 48: 1998-2006, 2008.

47. Chung HW, Lim JH, Kim MY, Shin SJ, Chung S, Choi BS, Kim HW, Kim YS, Park CW and Chang YS: High-fat diet-induced renal cell apoptosis and oxidative stress in spontaneously hypertensive rat are ameliorated by fenofibrate through the PPAR $\alpha$-FoxO3a-PGC-1 $\alpha$ pathway. Nephrol Dial Transplant 27: 2213-2225, 2012.

48. Geng T, Li P, Yin X and Yan Z: PGC-1 $\alpha$ promotes nitric oxide antioxidant defenses and inhibits FOXO signaling against cardiac cachexia in mice. Am J Pathol 178: 1738-1748, 2011.

49. Essers MA, de Vries-Smits LM, Barker N, Polderman PE, Burgering BM and Korswagen HC: Functional interaction between beta-catenin and FOXO in oxidative stress signaling. Science 308: 1181-1184, 2005.

50. Yang Y, Cehrke S, Imai Y, Huang Z, Ouyang Y, Wang JW, Yang L, Beal MF, Vogel H and Lu B: Mitochondrial pathology and muscle and dopaminergic neuron degeneration caused by inactivitation of Drosophila Pink1 is rescued by Parkin. Proc Natl Acad Sci USA 103: 10793-10798, 2006. 